\title{
Assessment of response to neoadjuvant radiochemotherapy with F-18 FLT and F-18 FDG PET/CT in patients with rectal cancer
}

\author{
Gundula Rendl • Lukas Rettenbacher • Johannes Holzmannhofer • \\ Lidwina Datz · Cornelia Hauser-Kronberger · Gerd Fastner · Dietmar Öfner • \\ Felix Sedlmayer $\cdot$ Christian Pirich
}

Received: 14 September 2014/Accepted: 26 November 2014/Published online: 11 December 2014

(C) The Japanese Society of Nuclear Medicine 2014

\begin{abstract}
Objective The comparison of 2-deoxy-2-[18F]fluoro-Dglucose (F-18 FDG) and $3^{\prime}$-deoxy-3'-[18F]fluorothymidine (F-18 FLT) imaging in patients with rectal cancer before and after neoadjuvant radiochemotherapy (RCT) in relation to histopathology and immunohistochemistry obtained from surgery.

Methods 20 consecutive patients $(15 \mathrm{~m}, 5 \mathrm{f})$, mean age of $65 \pm 10$ years were included into this prospective study with a mean follow-up of $4.1 \pm 0.8$ years.

Results Among histopathological responders $(n=8$ out of 20), posttreatment F-18 FLT and F-18 FDG scans were negative in $75 \%(n=6)$ and $38 \%(n=3)$, respectively. The mean response index (RI) was $61.0 \% \pm 14.0 \%$ for
\end{abstract}

EudraCT-Number: 2006-003828-11.

G. Rendl $(\square)$ - L. Rettenbacher · J. Holzmannhofer · C. Pirich Department of Nuclear Medicine and Endocrinology, Paracelsus Medical University Salzburg, Muellner Hauptstr. 48, 5020 Salzburg, Austria

e-mail: g.rendl@salk.at

L. Datz

Department of Radiology, Paracelsus Medical University

Salzburg, Salzburg, Austria

C. Hauser-Kronberger

Department of Pathology, Paracelsus Medical University

Salzburg, Salzburg, Austria

G. Fastner · F. Sedlmayer

Department of Radiotherapy and Radiooncology,

Paracelsus Medical University, Salzburg, Austria

D. Öfner

Department of Surgery, Paracelsus Medical University Salzburg,

Salzburg, Austria
F-18 FLT and $58.7 \% \pm 14.6 \%$ for F-18 FDG imaging. Peritumoral lymphocytic infiltration (CD3 positive cells) was significantly related to posttreatment $\mathrm{SUV}_{\max }$ in F-18 FDG but not F-18 FLT studies.

Conclusion A significant decrease of $\mathrm{SUV}_{\max }$ in F-18 FDG and F-18 FLT studies could be seen after RCT. Negative posttreatment F-18 FLT studies identified more histopathological responders.

Keywords $18 \mathrm{~F}-\mathrm{FDG} \cdot 18 \mathrm{~F}-\mathrm{FLT} \cdot \mathrm{PET} / \mathrm{CT} \cdot \mathrm{Rectal}$ cancer $\cdot$ Radiochemotherapy

\section{Introduction}

The assessment of response to neoadjuvant radiochemotherapy (RCT) in patients with rectal cancer is of potential clinical importance for the selection of the appropriate therapeutic strategy aiming for cure. This is achieved most commonly by surgery, but in selected cases with documented complete response to RCT, a wait-and-see strategy might be applied [1].

Many studies investigated the impact of imaging with positron emission tomography/computer tomography (PET/CT) using 2-deoxy-2-[18F]fluoro-D-glucose (F-18 FDG) to monitor the efficacy of RCT in rectal cancer. Some studies related long-term outcome to the course of F-18 FDG PET performed at baseline and about 2 weeks after start of therapy [2,3]. Other study designs employed F-18 FDG studies before and after RCT to define response criteria and relate them to long-term outcome [4, 5]. However, controversy persists about the reliability of F-18 FDG PET/CT to detect histopathological remission after RCT. An increased glucose metabolism of inflammatory tissues in F-18 FDG PET studies belongs to the potential 
confounders of reliable assessment of F-18 FDG imaging after RCT $[3,6]$.

$3^{\prime}$-deoxy-3'-[18F]fluorothymidine (F-18 FLT) has been repeatedly claimed as a more specific marker of tumor activity reflecting DNA synthesis and, therefore, tumor proliferation and growth. Numerous studies of F-18 FLT in animals and subsequent pilot patient studies have demonstrated that the tracer produces images of high contrast in both proliferating tissues and tumors [7-13], and F-18 FLT has been used in many different tumor entities to evaluate treatment response [14].There are some report about its accuracy in staging of colorectal cancer [15], but few related to monitoring of $\mathrm{RCT}[16,17]$.

The assessment of response to neoadjuvant radiochemotherapy in patients with rectal cancer is of particular clinical importance for the clinical management, since a wait-and-see strategy might be applied in patients with documented complete response.

The aim of this study was to evaluate and compare F-18 FDG and F-18 FLT PET/CT imaging in patients with rectal cancer before and after standardized RCT with respect to characterize responders and non-responders based on both histopathological and immunohistochemical criteria [18].

\section{Materials and methods}

\section{Patients}

In this prospective and single-center study, 22 consecutive patients were included, 15 male $(68 \%)$ and 7 female (32\%) patients with a mean age of $65 \pm 10$ years (range 41-83 years). Included were patients with biopsy-proven adenocarcinoma of the rectum; the tumor stage had to be cT3 or cT4 (cT3, $n=19,86 \%$; cT4, $n=3,14 \%$ ) (Table 1) and all patients had to be eligible for RCT due to tumor board decision.

All patients underwent the same study protocol, including conventional diagnostic evaluation using coloproctoscopy and abdominal CT before inclusion in the study. F-18 FDG and F-18 FLT PET/CT scanning were performed on two separate days at baseline before treatment and within 2-4 weeks after completion of the RCT protocol due to planned surgery soon after the completion of RCT.

This study was approved by the local Ethics Committee and all study participants gave written informed consent to their participation.

\section{F-18 FLT and F-18 FDG PET/CT imaging}

F-18 FLT PET/CT scans were obtained after fasting for a minimum of $6 \mathrm{~h}$ using a dedicated full ring PET/CT scanner (Biograph 6 PET/CT Tomograph, Siemens, Knoxville, Tennessee, USA). An intended activity of $4 \mathrm{MBq}$ F-18 FLT per kg body weight (minimum activity $300 \mathrm{MBq}$ ) was injected intravenously, and imaging was started 45-60 min post injection.

3-dimensional PET data acquisition was performed employing 6-8 bed positions ( $16.2 \mathrm{~cm}$ axial field of view) with a scan time of $3 \mathrm{~min}$ per bed position. The CT-based attenuation-corrected PET images were reconstructed with an iterative OSEM (ordered subset expectation maximization) algorithm (OSEM 2D, 4 iterations, 8 subsets) and smoothed with a Gaussian filter with $5 \mathrm{~mm}$ FWHM (Matrix $168 \times 168$, Voxelsize 4.06/4.06/5.0 mm).

Quality control on the PET/CT system was done according to the manufacturer guidelines and amended to local requirements. Cross-calibration was done twice a year and the measurements showed that semi-quantification in terms of $\mathrm{Bq} / \mathrm{ml}$ and $\mathrm{SUV}$, respectively, were stable over the whole life time of the system.

The reconstructed images were assessed on a diagnostic imaging computer monitor using axial, coronal and sagittal slicing. In addition to visual interpretation, semi-quantitative analysis using standardized uptake values (SUVs) were performed. Regions of interest (ROIs) were inserted according to the extent of the uptake of the lesion.

F-18 FDG PET/CT scans were obtained on the same full ring PET/CT scanner as F-18 FLT imaging studies. All patients were fasting for a minimum of $12 \mathrm{~h}$ prior to $\mathrm{F}-18$ FDG PET/CT imaging. Blood glucose levels were controlled in all patients before injection of F-18 FDG; none was higher than $120 \mathrm{mg} / \mathrm{dl}$. An intended activity of $4 \mathrm{MBq}$ F-18 FDG per kg body weight (minimum of $300 \mathrm{MBq}$ ) was injected intravenously, and imaging was started $60 \mathrm{~min}$ post injection.

Data acquisition and image reconstruction were conducted equally as in F-18 FLT PET/CT imaging (above). For radiotherapy planning, all patients were scanned in prone position (using the same belly-board as used during radiotherapy) for the staging $\mathrm{PET} / \mathrm{CT}$, however, follow-up PET/CT scanning was done in supine position. Afterwards, images were reformatted into supine position for image comparison and appraisal.

\section{CT imaging}

A low-dose computer tomography (130 kV, $30 \mathrm{mAS})$ was performed in all patients before PET scanning. CT data were used for attenuation correction of the PET data.

Interpretation of imaging studies

The PET study was interpreted by a board-certified Nuclear Medicine physician with more than 10 years of 
Table 1 Patient characteristics and clinical TNM-Stage
Tumor grading: all patients, except patient no. 17 (G3) had G2 tumors ( $G 2$ moderately differentiated/intermediate grade, $G 3$ poorly differentiated/ high grade)

$T$ primary tumor, $N$ local lymph node metastases, $M$ distant metastases

\begin{tabular}{llllllll}
\hline Patient no. & Age & Gender & T & N & M & Grading & Histology \\
\hline 1 & 50 & M & T3 & N0 & Mx & G2 & Adenocarcinoma (tubular-villous) \\
2 & 60 & M & T3 & N0 & Mx & G2 & Adenocarcinoma \\
3 & 57 & M & T4 & N0 & M0 & G2 & Adenocarcinoma \\
4 & 41 & F & T4 & N1 & M0 & G2 & Adenocarcinoma (mucinous) \\
5 & 74 & M & T3 & N0 & Mx & G2 & Adenocarcinoma \\
6 & 76 & F & T3 & N0 & M1 & G2 & Adenocarcinoma \\
7 & 55 & F & T3 & N0 & M0 & G2 & Adenocarcinoma \\
8 & 66 & M & T3 & N0 & Mx & G2 & Adenocarcinoma \\
9 & 67 & M & T3 & N1 & Mx & G2 & Adenocarcinoma \\
10 & 63 & M & T3 & N0 & M0 & G2 & Adenocarcinoma \\
11 & 74 & F & T3 & N0 & Mx & G2 & Adenocarcinoma \\
12 & 83 & M & T3 & N0 & Mx & G2 & Adenocarcinoma \\
13 & 81 & M & T3 & N0 & Mx & G2 & Adenocarcinoma (tubular) \\
14 & 73 & M & T4 & N0 & M0 & G2 & Adenocarcinoma \\
15 & 64 & F & T3 & N0 & Mx & G2 & Adenocarcinoma \\
16 & 61 & M & T3 & N0 & M0 & G2 & Adenocarcinoma \\
17 & 67 & M & T3 & N1 & M1 & G3 & Adenocarcinoma \\
18 & 65 & M & T3 & N0 & Mx & G2 & Adenocarcinoma \\
19 & 71 & F & T3 & N1 & M1 & G2 & Adenocarcinoma \\
20 & 69 & F & T3 & N0 & M0 & G2 & Adenocarcinoma \\
21 & 57 & M & T3 & N0 & Mx & G2 & Adenocarcinoma \\
22 & 57 & M & T3 & N1 & Mx & G2 & Adenocarcinoma \\
\hline
\end{tabular}

expertise. Qualitative (lesion, artifact) and semi-quantitative (SUV, as marker of tumor glucose metabolism for F-18 FDG and as marker of proliferation for F-18 FLT) evaluation of PET data were made for each patient on a Syngo workstation. If a focal area had increased tracer uptake, higher than the normal background (using the liver as reference in F-18 FDG studies, in F-18 FLT studies the abdominal background was used due to the higher liver uptake in FLT studies) activity, the lesion was considered positive for tumor tissue. If no significant focal uptake was found, the PET study was interpreted as negative. The maximal SUV of the lesion (marked by increased uptake) were documented in each patient.

F-18 FDG and F-18 FLT PET/CT data were compared before and after RCT, and SUV changes were evaluated and documented (no uptake/reduced uptake/equal uptake/ higher uptake than in the staging PET/CT). The mean absolute difference was calculated as absolute pretreatment $\mathrm{SUV}_{\text {max }}$ - posttreatment $\mathrm{SUV}_{\text {max }}$ difference (mean absolute difference $=\Delta \mathrm{SUV}$ ). The percentage change, named the mean response index (RI) was calculated using the following formula: $\mathrm{RI}=\left[\right.$ (pretreatment $\mathrm{SUV}_{\max }-$ posttreatment $\left.\mathrm{SUV}_{\max }\right) /$ pretreatment $\left.\mathrm{SUV}_{\max }\right] \times 100$.

Consensus readings with an experienced radiologist (more than 10 years of experience), who evaluated the CT data, were performed after each physician had reported their findings.

\section{Chemotherapy}

All patients received concomitant chemoradiation during the first 4 weeks of neoadjuvant chemotherapy with Capecitabine, plus weekly Oxaliplatin [19].

\section{Radiotherapy}

All patients received standardized three-dimensional conformal neoadjuvant radiation therapy for a duration of 5 weeks 5 times per week ( 25 fractions $\times 1.8$ Gray), with a tumor dose of 45 Gray. Target volume definition was carried out by hand and controlled using the PET component of both staging PET/CT [controlling if any uptake could be found outside the planning target volume (PTV)]. Radiation therapy was done using a isocentric 3-fieldtechniqe with one dorsal field and two lateral fields.

\section{Surgery}

All patients underwent resection with curative intent, performed at the latest 4 weeks after the end of the neoadjuvant radiochemotherapy. 
Histology

The resected tumor tissue was classified according to the TNM classification [20] and grading of the tumor differentiation was obtained. The resected tumor and the margins of the resectat were examined about tumor diameter, necrosis, fibrosis and inflammation.

The grading of tumor regression in response to neoadjuvant radiochemotherapy was based on the proportion of viable tumor tissue in relation to total tumor mass [18].

Grade of tumor regression induced by RCT was defined as follows: Grade 1a, complete ( $0 \%$ residual tumor) or Grade $1 \mathrm{~b}$, subtotal tumor regression $(<10 \%$ residual tumor per tumor bed); Grade 2, partial tumor regression (10-50\% residual tumor per tumor bed) and Grade 3, minimal or no tumor regression $(>50 \%$ residual tumor per tumor bed) [21].

Tumor regression grade (TRG) $1 \mathrm{a}$ and TRG $1 \mathrm{~b}$ were considered as indication for tumor response to therapy; whereas, TRG II and III were considered as indication for non-responding tumors.

Immunohistochemistry

The markers Ki-67, CD3 and p53 were investigated immunohistologically. $\mathrm{Ki}-67$ is used as proliferation marker, positive cells were scored as percentage of total amount of tumor cells. CD3 was used as a marker for T cell-mediated inflammation, and both peritumoral infiltration and overall infiltration of the surrounding tissue were analyzed.

Immunohistochemical staining for Pan-cytokeratin (AE1/3), CD3 and p53 was performed on routinely formalin-fixed paraffin embedded (FFPE) tissue, using a standardized automated platform (AutostainerPlus, Dako, DN) in combination with Envision polymer detection system (Dako, DN). AE1/3 antibody was used for detection of vital residual cancer cells in a final dilation of (1:400) and CD3 (1:200) for evaluation of the post therapeutic grade of inflammation. Residual vital cancer cells with genetic instability and a supposed more aggressive behavior were marked with p53 with a working dilution of $1: 200$.

3- $\mu$ m-thick, archival FFPE sections were deparaffinized with xylene, dehydrated, followed by heat-induced epitope retrieval (HIER) at $98{ }^{\circ} \mathrm{C}$ for $40 \mathrm{~min}$ in antigen retrieval buffer pH 9 (Dako, DN). Endogenous peroxidase blocking was carried out 10 min with $3 \% \mathrm{H}_{2} \mathrm{O}_{2}$ in absolute methanol and normal serum was applied. Primary antibodies and detection reagents were incubated at RT for $30 \mathrm{~min}$ and after several washes detection was performed using Envision detection system, followed by chromogenic visualization with diaminobenzidine (DAB). Nuclear counterstaining was performed with hematoxylin.
Statistical analysis

Numerical variables are given as mean \pm standard deviation (SD), and qualitative variables are reported as frequencies and percentages, respectively.

For statistical comparative analysis, the non-parametric Mann-Whitney Test, the $\chi^{2}$ Test and Spearman's Rho rank correlation coefficient were evaluated, using the program SPSS Statistics (Version 18.0).

\section{Results}

A total of 22 patients were included in the study, two of them were drop-outs due to study protocol violation; therefore, only 20 patients completed the study according to the protocol. Patient no. 6 underwent surgery directly after staging PET/CT and patient no. 7 had the staging PET/CT after the first course of RCT.

Mean activity for F-18 FDG PET/CT was $306 \pm 20 \mathrm{MBq}$ and $304 \pm 24 \mathrm{MBq}$ for pre- and posttreatment imaging studies, respectively. For F-18 FLT PET/CT imaging studies, the mean activity for pre- and posttreatment PET/CT was $301 \pm 14 \mathrm{MBq}$ and $307 \pm 20 \mathrm{MBq}$, respectively. All patients underwent both F-18 FDG and F-18 FLT PET/CT imaging studies within 14 days (pretreatment: mean time between FDG and FLT PET/CT $3 \pm 1$ days range 1-6 days; posttreatment: mean time between FDG and FLT PETCT $3 \pm 1$ days, range 1-4 days) before and after RCT with a mean time interval of $44 \pm 4$ days for F-18 FDG (median 43 days, range 37-54 days) and $44 \pm 4$ days for F-18 FLT (median 42 days, range 35-50 days), respectively. The mean time interval between the first staging PET/CT and surgery was $76 \pm 19$ days (range 65-105 days).

\section{Assessment of response by F-18 FDG and F-18 FLT PET/CT imaging}

\section{Visual analysis of the primary tumor}

Visually, only four out of 20 patients were categorized as negative in F-18 FDG PET/CT imaging (patient no. 3, 5, 15 and 16). In F-18 FLT PET/CT, 7 out of 20 patients were visually interpreted as positive, with reduced F-18 FLT uptake in the primary tumor after RCT [patient no. 1, 2, 11, 13, 14, 17 and 18; all other patients scans were classified as negative-for details see (Table 2)].

Compared to F-18 FDG (20\% of the repeated PET/CT scans were visually classified as negative regarding the primary tumor), more F-18 FLT PET/CT scans were negative $(65 \%)$. Notably, patient no. 19 (the only patient with immunohistochemical complete remission) had a negative 
Table $2 \mathrm{SUV}_{\max }$ of the primary tumor and response index (RI)

\begin{tabular}{|c|c|c|c|c|c|c|}
\hline \multicolumn{5}{|c|}{ Primary tumor-SUV $\mathrm{Smax}_{\max }$} & \multicolumn{2}{|c|}{$\begin{array}{l}\text { Response index } \\
\text { (RI) } \%\end{array}$} \\
\hline Patient no. & FDG 1 & FDG 2 & FLT 1 & FLT 2 & RI FDG & RI FLT \\
\hline 1 & 25.3 & 6.1 & 10.4 & 2.3 & 75.9 & 77.9 \\
\hline 2 & 22.1 & 7.1 & 7.9 & 3.8 & 67.9 & 51.9 \\
\hline 3 & 18.2 & 6.1 & 8.8 & 3.1 & 66.5 & 64.8 \\
\hline 4 & 25.8 & 7.3 & 8.4 & 2.3 & 71.7 & 72.6 \\
\hline 5 & 10.4 & 4.7 & 6.1 & 4.2 & 54.8 & 31.1 \\
\hline 6 & $(28.9)$ & - & $(6.3)$ & - & & \\
\hline 7 & $(11.4)$ & - & $(2.1)$ & - & & \\
\hline 8 & 15.6 & 8.1 & 6.8 & 3.1 & 48.1 & 54.4 \\
\hline 9 & 30.6 & 7.2 & 10.9 & 3.4 & 76.5 & 68.8 \\
\hline 10 & 22.6 & 6.7 & 7.7 & 2.5 & 70.4 & 67.5 \\
\hline 11 & 24.8 & 12.4 & 5.4 & 2.4 & 50.0 & 55.6 \\
\hline 12 & 18.8 & 6.5 & 7.6 & 2.5 & 65.4 & 67.1 \\
\hline 13 & 11.7 & 6.4 & 6.5 & 4.2 & 45.3 & 35.4 \\
\hline 14 & 20.9 & 8.8 & 8.7 & 4.5 & 57.9 & 48.3 \\
\hline 15 & 11.9 & 4.6 & 5.5 & 2.9 & 61.3 & 47.3 \\
\hline 16 & 14.7 & 7.3 & 7.3 & 3.2 & 50.3 & 56.2 \\
\hline 17 & 22.4 & 5.3 & 7.7 & 2.7 & 76.3 & 64.9 \\
\hline 18 & 14.8 & 8.6 & 12.7 & 6.3 & 41.9 & 50.4 \\
\hline 19 & 26.4 & 5.4 & 10.1 & 2.4 & 79.5 & 76.2 \\
\hline 20 & 12.9 & 6.8 & 11.3 & 2.8 & 47.3 & 75.2 \\
\hline 21 & 8.3 & 5.7 & 10.2 & 2.0 & 31.3 & 80.4 \\
\hline 22 & 9.1 & 5.9 & 7.8 & 2.1 & 35.2 & 73.1 \\
\hline
\end{tabular}

F-18 FDG/F-18 FLT 1 pretreatment PET/CT, F-18 FDG/F-18 FLT 2 posttreatment $\mathrm{PET} / \mathrm{CT}$, italic values represents patients without delineable F-18 FDG/F-18 FLT uptake in the primary tumors after RCT

F-18 FLT PET/CT scan after RCT; whereas, the primary tumor exhibited persistent tracer uptake in the respective F-18 FDG study (Table 2).

\section{Semi-quantitative analysis of the primary tumor}

The mean $\mathrm{SUV}_{\max }$ (maximal SUV of the lesion) of the primary tumor was $18.4 \pm 6.5$ for F-18 FDG (median 18.5, range 8.3-30.6) and $8.4 \pm 2.0$ for F-18 FLT PET-CT before therapy (median 7.9, range 5.5-12.7), respectively. After RCT, the $\mathrm{SUV}_{\max }$ of the primary tumor was significantly lowered to a mean max SUV of $6.9 \pm 1.7$ (median 6.6, range 4.6-12.4) and 3.1 \pm 1.0 (median 2.9, range 2.0-6.3) for F-18 FDG and F-18 FLT, respectively.

The mean RI index for F-18 FDG was $58.7 \pm 14.6 \%$ (median $59.6 \%$, range $31.3-79.5 \%$ ) and for F-18 FLT $61.0 \pm 14.0 \%$ (median $64.9 \%$, range $31.1-80.4 \%$ ) (Table 2).

The only patient with immunohistochemical complete remission (patient no. 19) had the highest RI in F-18 FDG and third highest in F-18 FLT (Fig. 1). However, for F-18
FDG no significant relation between RI versus (vs.) Ki-67 ( $p=0.613, r=0.124)$ as well as RI vs. $\mathrm{CD} 3(p=0.597$, $r=-0.129)$ could be found, respectively. RI vs. Ki-67 $(p=0.639, \quad r=-0.115) \quad$ as well as RI vs. CD3 $(p=0.675, r=0.676)$ showed no significant relation in F-18 FLT, too.

Lymph nodes 5 out of 20 patients had lymph node metastases in pretreatment F-18 FLT PET/CT $(n=5,3$ pararectal, 1 presacal and 1 iliacal), all of which could be found in F-18 FDG as well; however, in patient no. 19, F-18 FDG found two more lymph node metastases (3 pararectal, 2 presacal and 2 iliacal) than F-18 FLT. So, a total of 7 lymph node metastases were found in F-18 FDG imaging. In the pretreatment PET/CT studies, no uptake could be seen in inguinal lymph nodes.

After RCT, F-18 FDG PET/CT demonstrated visible uptake in inguinal lymph nodes in 3 patients: Patient no. 4 and no. 10 had uptake in bilateral inguinal lymph nodes, while patient no. 15 exhibited uptake in an inguinal rightsided lymph node (Fig. 2). In a fourth patient (no.9), F-18 FDG uptake was visible in an iliacal lymph node. In F-18 FLT PET/CT, uptake in 7 inguinal lymph nodes in 4 patients could be seen after RCT: Patient no. 4, 10 (same as F-18 FDG) and 15 showed uptake in bilateral lymph nodes (Fig. 2), and patient no. 8 demonstrated uptake in a leftsided inguinal lymph node.

The $\mathrm{SUV}_{\max }$ of lymph node metastases in F-18 FDG studies was $9.1 \pm 7.6$ (median 8.0, range 1.5-18.2) before RCT and $4.5 \pm 1.3$ (median 4.3, range 3.1-6.3) after RCT, respectively. In F-18 FLT imaging, the mean $\mathrm{SUV}_{\max }$ was $4.9 \pm 1.6$ (median 4.9, range 2.6-6.6) before RCT and $5.9 \pm 2.1$ (median 6.3, range 3.1-7.9) after RCT, respectively.

Liver uptake The mean $\mathrm{SUV}_{\max }$ of the liver was $3.3 \pm 0.9$ and $3.6 \pm 0.7$ for F-18 FDG PET/CT before and after RCT, respectively. For F-18 FLT PET/CT, the mean $\mathrm{SUV}_{\max }$ of the liver was higher with $7.7 \pm 1.0$ and $7.2 \pm 1.5$ before and after RCT, respectively.

Distant metastases Distant metastases were seen pretherapeutically in four patients with F-18 FDG (lung, $n=2$, liver, $n=4$, other, $n=1$ ), whereas no distant metastases could be detected by F-18 FLT PET/CT. The mean SUV $\mathrm{max}_{\text {max }}$ for distant metastases in F-18 FDG was $6.9 \pm 1.1$ (median 6.9, range 5.5-8.7). However, patient no. 6 underwent surgery immediately (without RCT), undergoing combined low anterior rectal resection and thoracotomy with histopathological verification of two lung metastases. Patient no. 16 had magnetic resonance imaging (MRI) showing the primary tumor and lymph node metastasis in the perirectal fatty tissue (histopathologically confirmed), all also seen in F-18 FDG PET/CT. In patient no. 17, two liver lesions in segment VIII could not be seen in the 

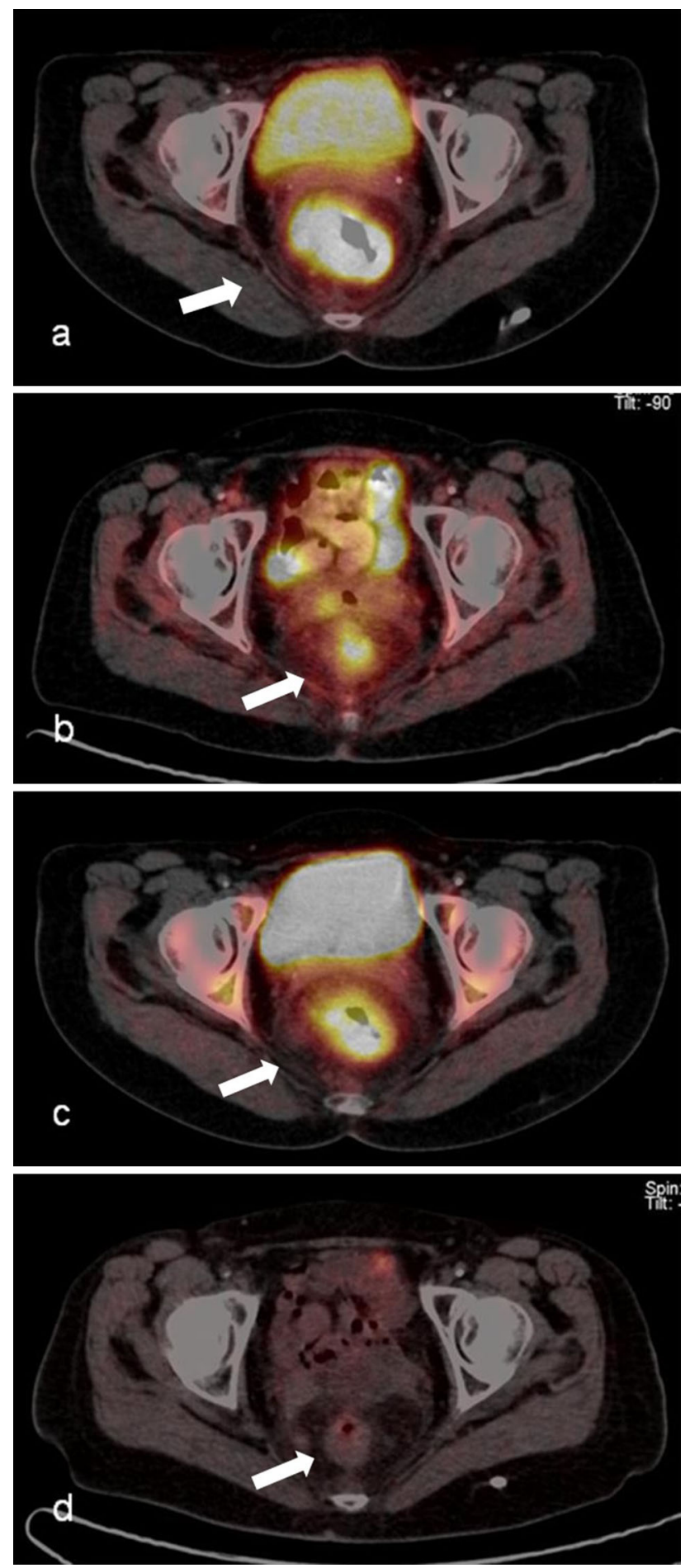

Fig. 1 Patient no. 19 (female, 71 years old), rectal adenocarcinoma, T3 N1 M1 at staging, complete remission after RCT in immunohistochemistry (a, b) F-18 FDG imaging study: a SUV max $_{26.4}$ before therapy, b SUV max 5.4 after therapy (c, d) F-18 FLT imaging study: c SUV $\mathrm{Sax}_{\max } 10.1$ before therapy, $\mathbf{d}$ after therapy no tumor uptake

follow-up F-18 FDG PET/CT after RCT, anymore, and no histopathological confirmation was made therefore. Patient no. 19, had 2 liver lesions in segment II not showing in the follow- up F-18 FDG PET/CT and underwent liver lesion resection nearly 1 year after therapy. In the histopathological work-up, liver metastases could be confirmed.

Histopathological analysis According to TNM criteria [20] for histopathological classification post RCT, 4 of the 20 patients $(20 \%)$ were classified as stage $0,6 / 20(30 \%)$ as stage I, 6/20 (30\%) as stage II and 4/20 (20\%) as stage III (Stage $0=\mathrm{T} 1-2$ N0 M0, Stage II A = T3 N0 M0, Stage II $B=$ T4 N0 M0, Stage III A $=$ T1-2 N1 M0, Stage III B $=$ T3-4 N1 M0, Stage III C = T1-4 N2 M0, Stage IV $=$ T1-4 N0-2 M1). In 11 cases (55\%), the tumor was downstaged histopathologically after surgery, including 4 patients (36\% of this subgroup, patient 1, 11, 15 and 19) with a complete histopathological response, while no downstaging was observed in 9 patients (45\%).

Of all 20 patients included in our study and submitted to surgery, 1 was classified as TRG 1a (5\%), 7 as TRG 1b (35\%), 5 as TRG $2(25 \%)$ and 6 as TRG $3(30 \%)$. One patient was not evaluated (Table 3 ).

Immunohistochemistry One patient (no. 19) exhibited complete immunohistochemical response, and two patients (no. 3 and 13) had partial immunohistochemical remission $(\mathrm{Ki} 67<10 \%)$. All other patients had Ki67 levels $>10 \%$ indicating clinically relevant tumor cell proliferation (Table 3; Fig. 1). Evidence of peritumoral T-cell infiltration after RCT was variable in our patient collective as was the degree of p53-positive tumor tissue (Fig. 3).

A significant correlation could be found between posttreatment F-18 FDG and CD3 infiltration $(p=0.031$, $r=0.471)$ Ki67 levels $(p=0.037, r=0.448)$ and p53 levels $(p=0.011, r=0.540)$. No relation was found between any RI for F-18 FLT or F-18 FDG PET/CT imaging and immunohistochemical markers.

Follow-up All patients but one (no. 22) are still alive up to now; however, 5 out of 20 patients developed distant metastases in follow-up.

Patient no. 8 showed a solitary lung lesion in the left upper lobe in a follow-up CT 2.6 years after initial diagnosis of rectal cancer. Pathohistology confirmed a lung metastasis of the known rectal cancer after surgical removal of the left upper lobe. In patient no. 9, a $3 \mathrm{~cm}$ lesion was seen in the right upper lobe in a follow-up F-18 FDG PET/CT 2.9 years after initial diagnosis; however, the patient was lost to follow-up. Recently, at a restaging F-18 FDG PET/CT 4.3 years after initial diagnosis, a progression of this lesion (now with a diameter of $5 \mathrm{~cm}$ ) was seen as well as a lymph node metastasis in the right hilus region. A bronchoscopy with biopsy confirmed the diagnosis of lung metastasis in the right upper lobe and lymph node metastasis. 
Fig. 2 Patient no. 15 (female, 64 years old), rectal adenocarcinoma, T3 N0 Mx, posttherapeutic PET/CT studies a F-18 FDG maximum intensity projection (MIP): F-18 FDG avid inguinal lymph node on the right side b F-18 FLT MIP: F-18 FLT avid bilateral inguinal lymph nodes
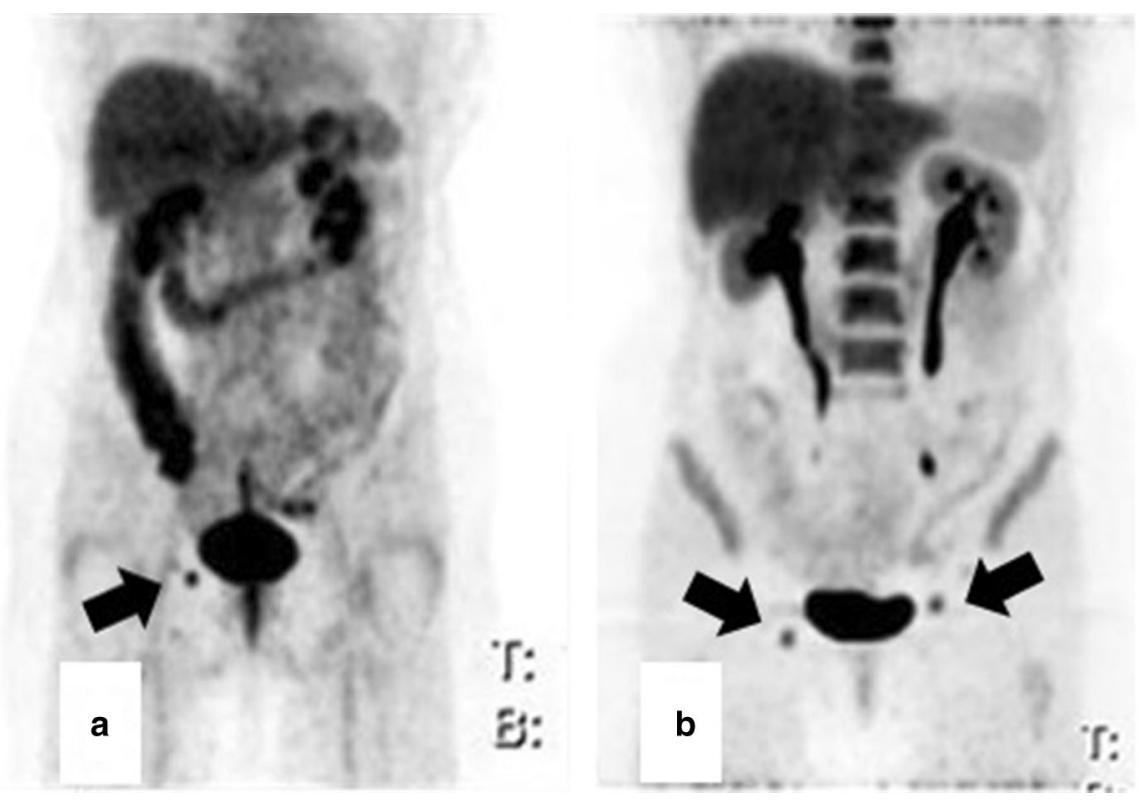

Table 3 Regression grade and immunohistochemical markers of the primary tumors after RCT

\begin{tabular}{|c|c|c|c|c|}
\hline $\begin{array}{l}\text { Patient } \\
\text { no. }\end{array}$ & $\begin{array}{l}\text { Regression } \\
\text { grade }\end{array}$ & $\begin{array}{l}\text { peritumoral CD3 } \\
(\%)\end{array}$ & $\begin{array}{l}\text { Ki67 } \\
(\%)\end{array}$ & P53 (\%) \\
\hline 1 & $\mathrm{Ib}$ & 25 & 12 & 0 \\
\hline 2 & III & 40 & 60 & 73 \\
\hline 3 & $\mathrm{Ib}$ & 25 & 8 & \\
\hline 4 & II & 46 & 56 & 7 \\
\hline 5 & $\mathrm{Ib}$ & 30 & 38 & 0 \\
\hline 6 & III & 40 & 47 & 5 \\
\hline 7 & II & 18 & 55 & 5 \\
\hline 8 & III & 17 & 58 & 68 \\
\hline 9 & $\mathrm{Ib}$ & 55 & 60 & 12 \\
\hline 10 & II & 5 & 35 & 10 \\
\hline 11 & III & 60 & 70 & 70 \\
\hline 12 & II & 7 & 25 & 3 \\
\hline 13 & $\mathrm{Ib}$ & 40 & 5 & 80 \\
\hline 14 & & 40 & 70 & 30 \\
\hline 15 & $\mathrm{Ib}$ & 33 & 55 & 0 \\
\hline 16 & II & 48 & 18 & 78 \\
\hline 17 & II & 25 & 38 & 0 \\
\hline 18 & III & 65 & 70 & 0 \\
\hline 19 & Ia & & $\begin{array}{l}\text { No } \\
\text { tumor }\end{array}$ & $\begin{array}{l}\text { No } \\
\text { tumor }\end{array}$ \\
\hline 20 & $\mathrm{Ib}$ & 63 & 33 & 20 \\
\hline 21 & III & 28 & 15 & 0 \\
\hline 22 & III & 33 & 61 & 55 \\
\hline
\end{tabular}

Patient no. 17 showed lung lesions in a follow-up F-18 FDG PET/CT nearly 1.3 years after initial diagnosis of rectal cancer. After surgical removal, pathohistology confirmed 3 lung metastases ( 2 in the left upper lobe and 1 in the left lower lobe). Another solitary lung lesion could be seen in the left lower lobe in a follow-up F-18 FDG PET/CT 2.8 years after initial diagnosis. Again, pathohistology confirmed a lung metastasis after resection.

Patient no. 18 showed a solitary lesion in the left lower lung lobe in a follow-up F-18 FDG PET/CT approximately 1.6 years after first diagnosis. Histology revealed a metastasis of the rectal cancer after resection of the lesion. A solitary lesion in the liver (segment III) was seen 2.3 years after initial diagnosis in CT and MRI (no histological confirmation up to now). In patient no. 19, a solitary liver lesion was found in segment II in a follow-up CT only 0.8 years after initial diagnosis. After resection of the left liver lobe, pathohistology confirmed a liver metastasis of the rectal cancer.

Patient no. 22 died 2.2 years after initial diagnosis due to a cardiac decompensation. No progression of disease could be seen in autopsy.

Mean overall survival in our patient collective was $4.1 \pm 0.8$ years (median 4.4 years, range $2.2-5.6$ ), with a mean time disease-free survival of $3.6 \pm 1.3$ years. No patient developed any local recurrence.

\section{Discussion}

Neoadjuvant treatment regimens have been reported to reduce local recurrence rates in rectal cancer [22, 23]. The assessment of response to RCT in patients with rectal cancer is of potential clinical importance for the therapeutic strategy that is most often surgery. Especially in 

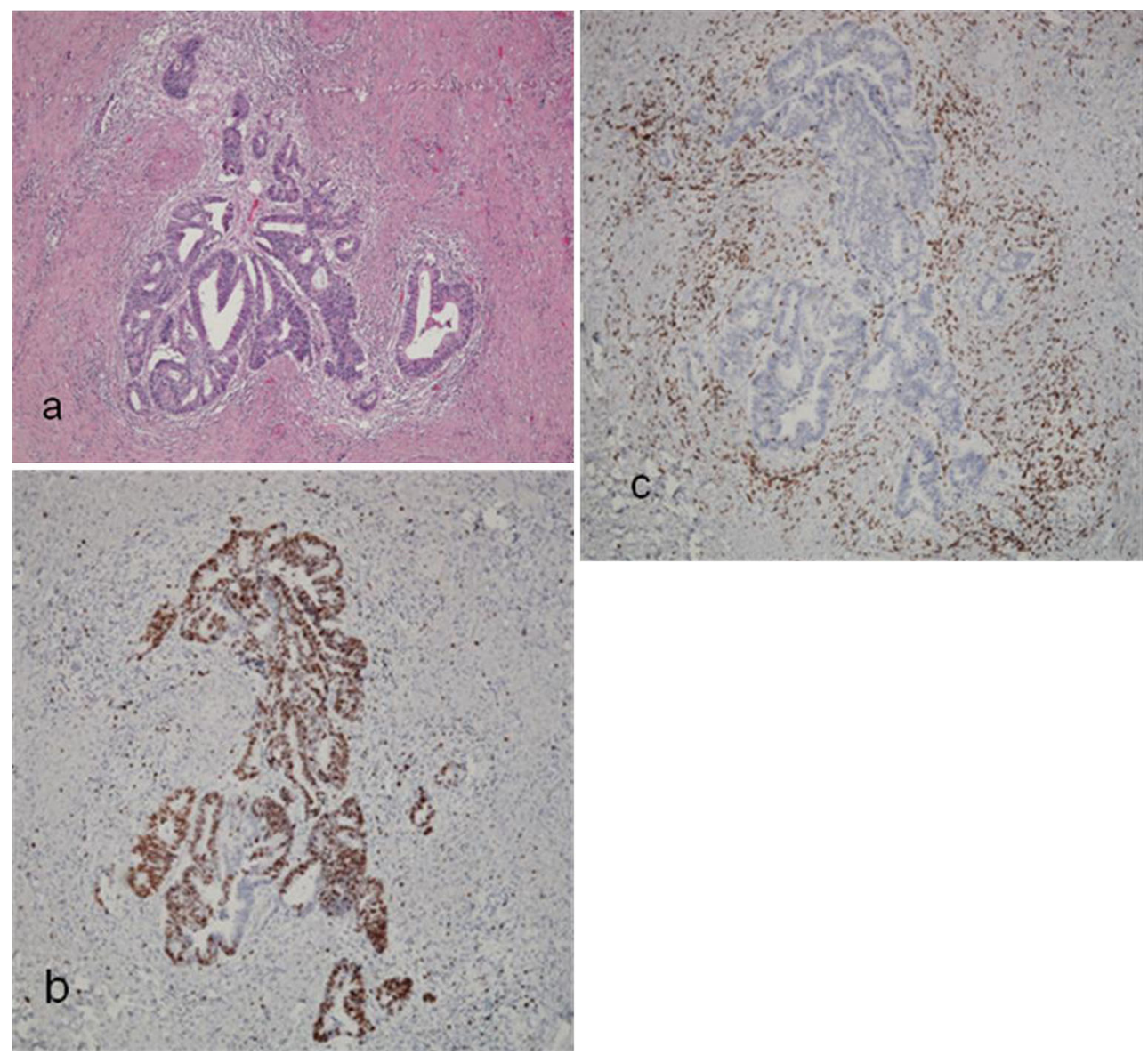

Fig. 3 Histology and immunohistochemistry of patient no. 4 with TRG II a hematoxylin-eosin stain b Ki-67 stain indicating proliferating cells c CD3 stain indicating peritumoral T-cell infiltration

distal rectal carcinomas, preoperative radiochemotherapy may offer potential to less extensive surgery, e.g., sphincter-preserving surgery with a better quality of life post surgically [22]. In selected cases, a wait-and-see strategy might be applied in patients with documented complete response [1]. Notably, in our study including T3 or T4 tumors only, no patient developed any locoregional tumor recurrence within 4 years of follow-up, which supports the effectiveness of the applied scheme of neoadjuvant RCT consisting of RT combined with capecitabine and oxaliplatin though no superiority to previous schemes had been reported [19].

For years, F-18 FLT PET has been claimed to be the most suitable tracer to indicate the persistence of viable tumor cells $[13,15,16]$ but few studies have been performed in rectal cancer patients [16, 17, 24]. To the best of our knowledge, our study is the largest one using a prospective design comparing F-18 FLT and F-18 FDG for the assessment of response after RCT.

This study confirms the limitations of FDG in monitoring neoadjuvant RCT by demonstrating that peritumoral
T-cell infiltration may be a significant confounder of SUV analysis after the end of fractionated radiotherapy [3, 6]. Thereby, it is noteworthy that a high CD3 score has been shown to be predictive of a longer colorectal cancer-specific survival [25]. In contrast, CD3 expression was not related to posttreatment F-18 FLT uptake, at all.

Notably, p53 tumor suppression gene activity and Ki-67 were also significantly related to posttreatment F-18 FDG levels, while no relation was seen in F-18 FLT studies. The inclusion of p53 tumor suppression gene activity and CD3 expression is a strength of our study showing the more complex impact of tumor biology on imaging parameters [26, 27].

Quantitative measures of changes in F-18 FDG or F-18 FLT uptake have been introduced and studied in rectal cancer to overcome the limitations of posttreatment visual interpretation of the scans [3-5]. Patients achieving histological remission (TRG $1 \mathrm{a}+1 \mathrm{~b}$ ) at the time surgery had a mean change in $\mathrm{SUV}_{\max }$ of $63 \%$ in F-18 FDG, and $60 \%$ in F-18 FLT studies; while non-responders (TRG $2+3$ ) exhibited a reduction in $\mathrm{SUV}_{\max }$ of 55 and $63 \%$ in F-18 FDG and F-18 FLT, respectively. In our patient collective 
these relative changes do not allow to discriminate responders from non-responders. A recent study [17] in 14 patients comparing F-18 FLT and F-18 FDG imaging found that F-18 FLT indicated histopathological response when achieving a decrease by at least $60 \%$ or a posttreatment $\mathrm{SUV}_{\text {max }}$ level below 2.2. We found just 2 patients with a posttreatment $\mathrm{SUV}_{\text {max }}$ in F-18 FLT imaging when applying these criteria. Both did not achieve total or near total histopathological remission. Eleven patients had a reduction of F-18 FLT SUV $>60 \%$. 5 of them were histopathological responders.

For F-18 FDG, numerous studies defined cut-off levels with an increased probability for histopathological response. A recent study of Calvo reported a $65 \%$ SUV cut-off 5 week after the end of radiotherapy [4]. Nine out of 20 patients exhibited a SUV decrease $\geq 65 \%, 4$ of them were histopathological responders.

To date, our data do not support the use of RI to determine histopathological responders eligible for a waitand-see strategy, since they could not be separated from minor or non-responders even when state-of-the-art schemes of neoadjuvant therapy were employed. Immunohistochemical findings might offer some explanations for this finding. While 8 patients were classified as histopathologic responders, this was only true for 3 by means of $\mathrm{Ki}$ 67 expression after RCT. In fact, $59 \%$ of the patients revealed a significant proportion of viable tumor cells.

In agreement with other studies, FLT uptake in the liver was higher than observed in FDG studies. However, no liver disease (such as hepatitis or others) was known in any patient, and ROIs in the liver in FDG studies were only used in liver segments without metastases.

Five patients in our collective developed progressive disease with distant metastasis, though locoregional tumor control had been achieved in all of them. Our data might support the concept of persisting tumor stem cells being responsible for the course of disease [28-30]. The amount of such viable tumor cells with potential for metastasis might be far below the detection limit of state-of-the art PET/CT scanners.

However, our study provides further evidence that inflammation might be an issue of relevance for the interpretation of both posttreatment F-18 FDG or F-18 FLT imaging.

After RCT, inguinal lymph nodes were seen in a few patients as reported before [31], These findings could be found either unilaterally or bilaterally. Most interestingly, the same inguinal lymph nodes could be seen in F-18 FDG and F-18 FLT PET/CT in 3 patients.

In the study of Perez et al. [31], the relevance of F-18 FDG positive inguinal lymph nodes in patients with rectal cancer after neoadjuvant chemoradiation was evaluated. They postulated that patients with persisting F-18 FDG uptake in inguinal lymph nodes had a worse prognosis. We could not support this hypothesis, since survival of our patients was favorable. Furthermore lymphatic drainage of rectal cancer cells into inguinal lymph nodes is a matter of controversy and rarely seen [32].

Two F-18 FLT PET/CT studies exhibited inguinal nodal uptake challenging the selectivity of F-18 FLT as a proliferation marker. Troost et al. [33] analyzed the value of F-18 FLT PET for determining the lymph node status in 10 patients with squamous cell carcinoma of the head and neck. Nine patients showed F-18 FLT PET-positive lymph nodes, but only 3 of these patients had histologically proven metastases. In the remaining 7 patients, an abundance of Ki-67 and iododeoxyuridine staining of B-lymphocytes in germinal centers of PET-positive lymph nodes could be seen, explaining the high rate of false-positive findings.

Semi-quantification is essential in therapy monitoring. In small lesion, i.e., lymph node metastasis, SUV measurements are biased by the partial-volume effect mainly caused by the limited spatial resolution of PET detectors and image sampling.

Among many parameters $\mathrm{SUV}_{\max }$ has been shown to reflect reliably maximum glucose metabolism in tumor tissue in F-18 FDG studies and maximum proliferation rate in F-18 FLT studies [34]. In accordance with international guidelines we employed the $\mathrm{SUV}_{\max }$ for the measurement of tracer uptake being reproduced reliably as evidenced by cross-calibration data. However, this is only one important aspect of standardization in (multi-center) clinical trials which are defined in current EANM procedure guidelines [34, 35].

Limitations of the study: Only a small number of patients were enrolled in this study, due to the complicated immunohistochemical work-up. Another limitation of the study is that none of the inguinal lymph nodes (either unilateral or bilateral) seen post therapeutically was resected. However, no patient presented with inguinal lymph node metastasis or local recurrence during a mean follow-up period of 4.1 years. The clinical course makes it very likely that the increased F-18 FLT uptake after RCT in inguinal lymph nodes can be related to an unspecific inflammatory reaction to radiation therapy.

Notably, F-18 FLT PET/CT was still able to detect lymph node metastases in our patients. However, all lymph node metastases could be seen with F-18 FDG PET/CT, as well. F-18 FDG PET/CT imaging even revealed two more lymph node metastases in one patient.

\section{Conclusion}

In summary, clinical response to RCT with capecitabine, plus weekly oxaliplatin is commonly observed in rectal 
cancer patients and associated with a significant decrease of $\mathrm{SUV}_{\max }$ in both F-18 FDG and F-18 FLT studies. However, in our study both F-18 FLT and F-18 FDG PET/ CT imaging did not reliably separate patients with histopathological response (TRG 1a and b) and immunohistochemical remission (percentage of $\mathrm{Ki}-67$ positive cells $<10 \%$ ) from those with incomplete response. Thus, this study does not support the use of single F-18 FLT or F-18 FDG SUV cut-off values for the definition of response. Two weeks after RCT, the degree of peritumoral lymphocyte infiltration as given by CD3 expression had significant impact on posttreatment F-18 FDG levels, as did Ki67 and p53 activity.

Future multi-center studies should address the definition of complete responders by F-18 FLT and F-18 FDG imaging under consideration of these important factors affecting tumor biology and response to RCT. They must be adequately powered since even advanced schemes of RCT results in relatively low rates of complete responders, particularly if defined by both histopathology and immunohistochemistry.

Conflict of interest The authors declare that they have no conflict of interest.

\section{References}

1. Maas M, Beets-Tan RG, Lambregts DM, Lammering G, Nelemans PJ, Engelen SM, et al. Wait-and-see policy for clinical complete responders after chemoradiation for rectal cancer. J Clin Oncol. 2011;29:4633-40.

2. Avallone A, Aloj L, Caracó C, Delrio P, Pecori B, Tatangelo F, et al. Early FDG PET response assessment of preoperative radiochemotherapy in locally advanced rectal cancer: correlation with long-term outcome. Eur $\mathrm{J}$ Nucl Med Mol Imaging. 2012;39:1848-57.

3. Janssen MH, Öllers MC, van Stiphout R, Riedl RG, van den Bogaard J, Buijsen J, et al. PET-based treatment response evaluation in rectal cancer: prediction and validation. Int $\mathrm{J}$ Radiat Oncol Biol Phys. 2012;82:871-6.

4. Calvo FA, Sole CV, de la Mata D, Cabezón L, Gómez-Espí M, Alvarez E, et al. 18F-FDG PET/CT-based treatment response evaluation in locally advanced rectal cancer: a prospective validation of long-term outcomes. Eur J Nucl Med Mol Imaging. 2013;40:657-67.

5. Murcia Duréndez MJ, Frutos Esteban L, Luján J, Frutos MD, Valero G, Navarro Fernández JL, et al. The Value of 18F-FDG PET/CT for assessing the response to neoadjuvant therapy in locally advanced rectal cancer. Eur J Nucl Med Mol Imaging. 2013;40:91-7.

6. Shreve PD, Anzai Y, Wahl RL, Shreve PD, Anzai Y, Wahl RL. Pitfalls in oncologic diagnosis with FDG PET imaging: physiologic and benign variants. Radiographics. 1999;19:61-77.

7. Shields AF. Positron emission tomography measurement of tumor metabolism and growth: its expanding role in Oncology. Mol Imaging Biol. 2006;8:141-50.

8. Shields AF, Grierson JR, Dohmen BM, Machulla HJ, Stayanoff JC, Lawhorn-Crews JM, et al. Imaging proliferation in vivo with
[F-18]FLT and positron emission tomography. Nat Med. 1998;4:1334-6.

9. Vesselle H, Grierson J, Muzi M, Pugsley JM, Schmidt RA, Rabinowitz $\mathrm{P}$, et al. In vivo validation of $3^{\prime}$ deoxy-3'-[(18)F]fluorothymidine $([(18) \mathrm{F}] \mathrm{FLT})$ as a proliferation imaging tracer in humans: correlation of [(18)F]FLT uptake by positron emission tomography with Ki-67 immunohistochemistry and flow cytometry in human lung tumors. Clin Cancer Res. 2002;8:3315-23.

10. van Waarde A, Cobben DC, Suurmeijer AJ, Maas B, Vaalburg W, de Vries EF, et al. Selectivity of F-18-FLT and F-18-FDG for differentiating tumor from inflammation in a rodent model. J Nucl Med. 2004;45:695-700.

11. van Waarde A, Jager PL, Ishiwata K, Dierckx RA, Elsinga PH. Comparison of sigma-ligands and metabolic PET tracers for differentiating tumor from inflammation. J Nucl Med. 2006;47: $150-4$.

12. Barthel H, Cleij MC, Collingridge DR, Hutchinson OC, Osman S, $\mathrm{He} \mathrm{Q}$, et al. $3^{\prime}$-deoxy- $3^{\prime}$-[18F]fluorothymidine as a new marker for monitoring tumor response to antiproliferative therapy in vivo with positron emission tomography. Cancer Res. 2003;63: 3791-8.

13. Dittmann H, Dohmen BM, Kehlbach R, Bartusek G, Pritzkow M, Sarbia M, et al. Early changes in $\left[{ }^{18}\right.$ F]FLT uptake after chemotherapy: an experimental study. Eur J Nucl Med. 2002;29: 1462-9.

14. Sanghera B, Wong WL, Sonoda LI, Beynon G, Makris A, Woolf $\mathrm{D}$, et al. FLT PET-CT in evaluation of treatment response. Indian J Nucl Med. 2014;29:65-73.

15. Francis DL, Visvikis D, Costa DC, Arulampalam TH, Townsend C, Luthra SK, et al. Potential impact of $[18 \mathrm{~F}] 3^{\prime}$-deoxy-3'-fluorothymidine versus [18F]fluoro-2-deoxy-D-glucose in positron emission tomography for colorectal cancer. Eur J Nucl Med Mol Imaging. 2003;30:988-94.

16. Wieder HA, Geinitz H, Rosenberg R, Lordick F, Bekcer K, Stahl A, et al. PET imaging with $[18 \mathrm{~F}] 3^{\prime}$-deoxy- $3^{\prime}$-fluorothymidine for prediction of response to neoadjuvant treatment in patients with rectal cancer. Eur J Nucl Med Mol Imaging. 2007;34:878-83.

17. Dehdashti F, Grigsby PW, Myerson RJ, Nalbantoglu I, Ma C, Siegel BA. Positron emission tomography with [(18)F]-3'-deoxy$3^{\prime}$ fluorothymidine (FLT) as a predictor of outcome in patients with locally advanced resectable rectal cancer: a pilot study. Mol Imaging Biol. 2013;15:106-13.

18. Becker K, Müller JD, Schulmacher C, Ott K, Fink U, Busch R, et al. Histomorphology and grading of regression in gastric carcinoma treated with neoadjuvant chemotherapy. Cancer. 2003;98: 1521-30.

19. Öfner D, Devries AF, Schaberl-Moser R, Greil R, Rabl H, Tschmelitsch J, et al. Preoperative oxaliplatin, capecitabine, and external beam radiotherapy in patients with newly diagnosed, primary operable, cT3NxM0, low rectal cancer. Strahlenth Onkol. 2011;187:100-7.

20. Edge S, Byrd DR, Compton CC, Fritz AG, Greene FL, Trotti A, editors. AJCC Cancer staging Manual. 7th ed. New York: Springer; 2010.

21. Kim CJ, Yeatman TJ, Coppola D, Trotti A, Williams B, Barthel JS, et al. Local excision of T2 and T3 rectal cancers after downstaging chemoradiation. Ann Surg. 2001;234:352-8.

22. Medical Research Council Rectal Cancer Working Party. Randomised trial of surgery alone versus radiotherapy followed by surgery for potentially operable locally advanced rectal cancer. Lancet. 1996;348:1605-10.

23. Julien LA, Thorson AG. Current neoadjuvant strategies in rectal cancer. J Surg Oncol. 2010;104:321-6.

24. Yamamoto Y, Kameyama R, Izuishi K, Takebayashi R, Hagiike $\mathrm{M}$, Asakura M, et al. Detection of colorectal cancer using $18 \mathrm{~F}$ - 
FLT PET: comparison with 18F-FDG PET. Nucl Med Commun. 2009;30:841-5.

25. Dahnlin AM, Henriksson ML, Van Guelpen B, Stenling R, Öberg A, Rutegard J, et al. Colorectal cancer prognosis depends on T-cell infiltration and molecular characteristics of the tumor. Mod Pathol. 2011;24:671-82.

26. Chalkidou A. Landau DB, Odell EW, Cornelius VR, O`Doherty MJ, Marsden PK. Correlation between Ki-67 immunohistochemistry and $18 \mathrm{~F}$-fluorothymidine uptake in patients with cancer: a systematic review and meta-analysis. Eur J Cancer. 2012;48:3499-513.

27. Riedl CC, Akhurst T, Larson S, Stanziale SF, Tuorto S, Bhargva A, et al. 18F-FDG PET scanning correlates with tissue markers of poor prognosis and predicts mortality for patients after liver resection for colorectal metastases. J Nucl Med. 2007;48:771-5.

28. Jang J, Yue JB, Liu J, Yu JM. Repopulation of tumor cells during fractionated radiotherapy and detection methods. Oncol Lett. 2014;7:1755-60.

29. Ishii H, Doki Y, Mori M. Perspective beyond cancer genomics: bioenergetics of cancer stem cells. Yonsei Med J. 2010;51: $617-21$.

30. Stelzner F, von Mallek D, Ruhlmann J, Biersack HJ. PET-CT studies of metastasizing cancer of the colon and rectum.
Variability of tumor aggressiveness as a micro-evolutionary process of cancer stem cells with predetermined prognosis. Chirurg. 2009;80:645-51 German.

31. Perez RO, Habr-Gama A, Sao Juliao GP, Proscurshim I, Ono CR, Lynn P, et al. Clinical relevance of PET/CT positive inguinal lymph nodes in rectal cancer after neoadjuvant chemoradiation. Colorectal Dis. 2013;15:674-82.

32. Tocchi A, Lepre L, Costa G, Liotta G, Mazzoni G, Agostini N, et al. Rectal cancer and inguinal metastases: prognostic role and therapeutic indications. Dis Colon Rectum. 1999;42:1464-6.

33. Troost EG, Vogel WV, Merkx MA, Slootweg PJ, Marres HA, Peeters WJ, et al. 18F-FLT PET does not discriminate between reactive and metastatic lymph nodes in primary head and neck cancer patients. J Nucl Med. 2007;48:726-35.

34. Vanderhoek M, Perlman SB, Jeraj R. Impact of different standardized uptake value measurements on PET-based quantification of treatment response. J Nucl Med. 2013;54:1188-94.

35. Boellard R, O'Doherty MJ, Weber WA, Mottaghy FM, Lonsdale MN, Stroobants SG, et al. FDG PET and PET/CT: EANM procedure guidelines for tumour PET imaging: version1.0. Eur J Nucl Med Mol Imaging. 2010;37:181-200. 